\title{
Development of a Hybrid Field/Zone Fire Model
}

\author{
DANIEL BURTON, ANGUS GRANDISON, MAYUR PATEL, EDWIN GALEA, and JOHN EWER \\ Fire Safety Engineering Group \\ The University of Greenwich \\ 30 Park Row, Greenwich, London SE10 9LS, UK
}

\begin{abstract}
A novel hybrid field/zone fire model, coupling the SMARTFIRE CFD fire model to both the CFAST zone model and a custom zone model is presented. The intention of the hybrid model is to reduce the computational overheads incurred in using fire field models to simulate large geometries such as large buildings or large passenger ships, while maintaining the accuracy of the fire field model. In using the hybrid model, only the most important parts of the geometry are fully modeled using the field model. Other less important parts of the geometry are modeled using the zone model. From the field model's perspective, the zone model is used to represent parts of the geometry as an accurate boundary condition. By using this approach, many computational cells are replaced by a simple zone model, saving computational costs. Two tests cases demonstrating the technique are presented. It is shown that the hybrid approach is capable of producing reasonably accurate predictions of fire development while substantially reducing computational costs. It is shown that by removing some $56 \%$ of the CFD solution domain, the hybrid case can achieve a saving of $48 \%$ in the run time.
\end{abstract}

KEYWORDS: modeling, compartment fires, CFD, zone models, hybrid, simulation.

\section{NOMENCLATURE LISTING}

$\begin{array}{llll}C_{P} & \text { specific heat, constant pressure }\left(\mathrm{J} \cdot \mathrm{kg}^{-1} \cdot \mathrm{K}^{-1}\right) & V & \text { volume }\left(\mathrm{m}^{3}\right) \\ C_{V} & \text { specific heat, constant volume }\left(\mathrm{J} \cdot \mathrm{kg}^{-1} \cdot \mathrm{K}^{-1}\right) & v & \text { velocity }\left(\mathrm{m} \cdot \mathrm{s}^{-1}\right) \\ g & \text { acceleration due to gravity }\left(\mathrm{m} \cdot \mathrm{s}^{-2}\right) & \text { Greek } & \\ h & \text { enthalpy }(\mathrm{J}) & \Gamma \quad \text { effective exchange coefficient } \\ I & \text { interface height }(\mathrm{m}) & \gamma & \text { ratio of specific heats }\left(C_{P} / C_{V}\right) \\ m & \text { mass }(\mathrm{kg}) & \rho & \text { density }\left(\mathrm{kg} \cdot \mathrm{m}^{-3}\right) \\ P & \text { pressure }(\mathrm{Pa}) & \Phi \text { fluid field variable } \\ R & \text { gas constant }\left(\mathrm{m}^{2} \cdot \mathrm{K}^{-1} \cdot \mathrm{s}^{-2}\right) & \text { subscripts } \\ S & \text { source term } & u \quad \text { upper zone layer } \\ T & \text { temperature }(\mathrm{K}) & l & \text { lower zone layer } \\ t & \text { time }(\mathrm{s}) & r \text { reference or ambient condition }\end{array}$

\section{INTRODUCTION}

The use of computational fluid dynamics (CFD) based fire field modeling has become increasingly popular over the past twenty years and has been used in a number of different scenarios [1-4]. One of the major disadvantages of fire field modeling is the time required to run the models [5]. Parallel processing is one way of reducing run times associated with running fire models [6], and while successful, many engineers may not have access to more than one or two computers. Another methodology for reducing runtime is to make use of advanced solver technology such as group solvers [7]. Using this approach cells/regions are combined into logical groups, making it possible to significantly reduce computational requirements by setting solver criteria on a group-by-group basis. In this way, regions requiring less computation can be lowered in priority, allowing computational effort to be focused as required. In this paper we explore a novel methodology which combines the CFD fire field model approach to that of the zone modeling approach [11] within the simulation environment to produce a hybrid modeling methodology. Within the hybrid approach, areas of the solution domain that would normally be modeled using the expensive field modeling approach are replaced with a zone model representation. Replacing the field model with a zone model in appropriate parts of the geometry could lead to a considerable saving in run time, whilst maintaining the accuracy of the simulations within the relevant portions of the solution domain. The hybrid approach would be particularly suited to larger domains containing numerous small compartments where 
large speed ups in solution time could be realized. Large passenger ships such as modern cruise ships are one example of such environments. The work described in this paper forms part of the EU Framework 7 project FIREPROOF, which is investigating the use of fire modeling for large passenger ships. The hybrid modeling technology described in this paper is being developed to reduce the run time associated with detailed fire simulations required for risk assessment analysis of passenger ship designs. Previous work on the implementation of hybrid models focused initially on two-dimensional problem domains [8]; this was expanded to three dimensions [9], and later work allowed for simulations across different floors of a multifloor domain [10]. The hybrid model proposed here utilizes the zone model representation to replace the field calculation in relatively small compartments not directly involved in the scenario, or regions of large solution domains which are far from the region of fire origin and of little direct interest. The hybrid model utilizes two approaches, the first approach couples the CFAST [11] zone model with the SMARTFIRE $[3,4,12]$ CFD fire model; the second approach couples a custom zone model with the SMARTFIRE CFD fire model. An earlier version of the hybrid model presented here was discussed in a previous paper [13]. In this earlier work the hybrid approach was restricted to compartments in which the zone model component was open to the outside and utilized the CFAST zone model. In the current implementation this restriction has been lifted allowing zone model compartments with no external vent of their own. This was achieved via the implementation of a custom zone model which is the focus of this paper. In this case, zone pressure release must occur through the CFD domain. In addition, hybrid simulation consisting of multiple zone rooms independently connected to the CFD domain is now addressed.

\section{FIRE MODELING APPROACHES}

\section{Zone Modeling}

Computer-based zone models were first developed in the early seventies and have been used extensively over the years in modeling a large catalogue of fire scenarios [14-16]. The underlying assumption of zone models is that a room can be divided into a number of distinct horizontal zones or layers, and the temperature, density and other attributes (e.g. product concentrations) are assumed to be uniform within each layer at any point in time, i.e. the layers are fully mixed. Experimental fire data suggests that fire gases stratify into these distinct layers, and while these values are never in reality uniform the variations through the layer, compared with those between the layers, are small enough to be assumed negligible. Due to stratification between the existing ambient 'cold' air and the fire affected 'hot' gases, a two layer zone model is generally accepted as a valid assumption allowing the prediction of reasonably accurate layer temperatures and the interface height. The mathematical model describing the zone model consists of a set of ordinary differential equations derived from conservation equations of mass and energy.

Zone models continue to be popular and effort is being invested to improve zone modeling capabilities; for example Li and Chow [17] have developed a water suppression capability within a single-zone zone model, Konecki and Pólka [18] have developed complex species transfer mechanisms, and Chen et al. [19] have developed zone models which make use of multiple layers (>2) within a single compartment. The main advantage of zone modeling is the comparatively small computational requirements compared to CFD models. Zone model calculations typically require only minutes rather than the many hours associated with CFD fire modeling.

\section{Fire Field Modeling}

Field modeling is more computationally demanding than its zone modeling counterpart, and is based on the more fundamental physics of the fluid flow [20]. Due to its reduced reliance on empiricism, the range of applicability is generally far greater for the field modeling approach compared to that of the zone model. In fire field modeling, the fluid flow is governed by a set of three-dimensional partial differential equations. This set consists of the continuity equation, the momentum equations in three space dimensions, the energy equation, the user equations for mass and mixture fraction, and the equations for the turbulence model; in this case the $k-\varepsilon$ model which incorporates buoyancy modification. The generalized governing equation for all variables is expressed in the form shown in Eq. 1, 


$$
\frac{\partial \rho \phi}{\partial t}+\nabla(\rho \bar{U} \phi)=\nabla\left(\Gamma_{\phi} \nabla \phi\right)+S_{\phi}
$$

This collection of partial differential equations is solved numerically, usually using iterative methods [20].

\section{The Hybrid Model}

The basic premise of the hybrid model is to combine the use of the two fire modeling approaches to benefit from each of the models' strong points whilst minimizing their respective disadvantages. The CFD model would be used primarily in compartments where accuracy is essential, such as the room of fire origin and regions where detailed analysis is required. It would also be used in compartments not adequately represented by zone models, such as long corridors or tall compartments. Finally, the CFD model would also be used in compartments containing complex flow qualities, such as strong turbulence and curl of the velocity field, which can have a significant effect on the simulation. The zone model would then be used in the remaining compartments.

Use of the zone model within these compartments allows parameters such as layer height and average temperature to be determined. This information is extremely useful when performing evacuation simulation or risk analysis. The real strength of the hybrid model is realized in situations containing many such compartments, each of which may be insignificant in isolation but together having a very large cumulative effect. Environments especially suited to hybrid treatment are large domains with numerous small compartments such as hotels, prisons and passenger ships.

The majority of the research effort involved in developing hybrid field/zone models focuses on the interface connecting the two separate models. These models have been individually validated, therefore any errors or inconsistencies will stem from how the interface is handled, both in the way it is represented within the models themselves and in how calculations and conversions are performed across it.

\section{THE HYBRID MODEL IMPLEMENTATION}

Within the field model portion of the hybrid model, the interface is modeled as a dynamic fixed pressure and temperature boundary condition, the values for which are obtained from the zone model results. This is performed by enhancing the code's existing treatment of fixed-pressure boundary conditions, by allowing variation of pressure, temperature and density across the interface (see Fig. 1). Within the zone model the interface will not be visible per-se nor modeled along with its internal connections, but will simply be implemented by creating a source/sink term in each layer to represent the net flow between the models.

The pressure for the boundary condition is calculated from a hydrostatic pressure distribution similar to the treatment of pressure within the zone model itself; the applied temperatures and densities are calculated based on flow direction and height. When these values are applied on the boundary condition, and SMARTFIRE is run for a single iteration, a flow is calculated across the interface. It is from this flow that the summed values to be communicated back to the zone model are found. At each cell-face lying on the boundary, the mass and enthalpy fluxes across the interface are calculated. These fluxes are grouped depending on which layer of the zone model they are depositing/extracting from, and are then applied to the appropriate layers as source/sink terms respectively.

In both directions there is an issue of converting the different forms of data that the respective models use so that they can interact correctly. The field model provides as many sets of data as there are cells neighboring the boundary condition in question (typically around 50-200 cells), whereas the zone model simply has two sets of values belonging to the upper and lower layers. When communicating data from SMARTFIRE to the zone model, a simple sum is used to reduce the resolution of the data to values for each of the two layers. In the reverse direction there is the issue of taking the sparse data of the zone model and applying it to the numerous CFD cells on the interface. 


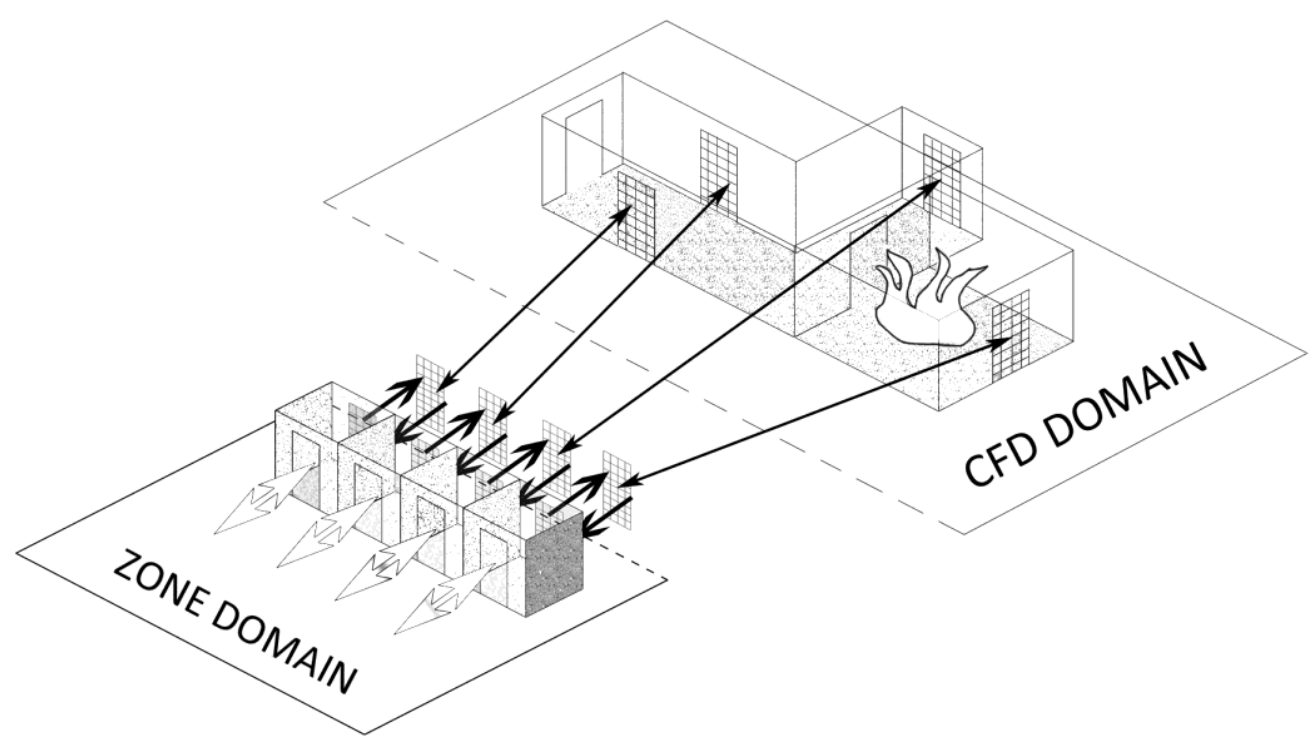

Fig. 1. Replacing CFD compartments with zone model representations.

Passing fluxes from a CFD to a zone model (and pressures from zone to CFD) ensures conservation across the interface; if instead pressure was passed from SMARTFIRE the calculation of fluxes for conversion to layer sources would have to be performed within the zone model itself. The zone model calculation for fluxes is fundamentally different to that of SMARTFIRE, and would result in discrepancies between the net flux leaving the CFD domain at the boundary and the net flux being accounted for by the sources within the zone model. With differing representation of fluxes it would be possible to create and destroy both enthalpy and mass across the interface, invalidating the very conservation principle the two models are based on.

\section{Pressure Boundary Condition}

The hybrid interface is represented within SMARTFIRE as a pressure boundary condition having variation in the vertical direction. Along with a value for pressure, accurate treatment of the hybrid interface also requires temperature and density to be declared at the boundary condition. These values are used to address convected quantities for flow entering the CFD domain from the zone model; the temperature values can also contribute to diffusion at the interface.

The value of pressure assigned at any point on this interface consists of three components: the room pressure supplied by the zone model, a hydrostatic term representing changes in pressure for varying height and density, and a dynamic term representing the variation in pressure over the hydrostatic value for flow speed.

$$
\begin{aligned}
& P_{\text {room }}=P_{\text {zone }} \\
& P_{\text {static }}=\left\{\begin{array}{c}
-g \rho_{l} h \quad: h \leq I \\
-g\left(\rho_{l} l+\rho_{u}(h-I)\right): \\
h>I
\end{array}\right. \\
& P_{\text {dynam }}=-\frac{\rho v^{2}}{2}
\end{aligned}
$$

where $v$ is the component of velocity normal to the interface.

The first component of room pressure (Eq. 2) corresponds to the total enthalpy contained within a zone compartment and represents the notion of a room being pressurized. Because the hydrostatic pressure manifests as a reduction with increasing height, this floor pressure is the highest value attained within the 
room. In this way it forms the basis for comparisons between rooms and differences in this value are the main driving force of flow. The zone model lacks any variation in pressure throughout the rooms but the consequences of layers of differing depth, temperature and density need to be accounted for. Due to the absence of momentum and velocities, dynamic pressure is not represented within the zone model itself and a standard hydrostatic treatment is used; this treatment is extended to the interface through the second pressure component (see Eq. 3). Despite the absence of momentum and dynamic pressure within the zone model, accurate treatment at the interface still requires inclusion of this term. This is addressed through the final component which is a pressure drop term representing the dynamic variation in pressure, due to flow speed, over the zone hydrostatic pressure where flow is assumed to have come to a state of rest (see Eq. 4). This drop term represents the pressure observed at a point on a streamline that comes to rest at the zone pressure value, and it accounts for the pressure gradient applying over the length of a streamline, not instantaneously at a point.

Calculating the values of temperature and density to apply to the boundary condition in SMARTFIRE is a straightforward matter. For flow from CFD to zone, these values are obtained directly from the boundary cell at which the flow leaves the CFD domain. For flow from zone to CFD, the values of temperature and density at a face are taken from the layers in which the flow originates. Flow is assumed to have originated from the layer that shares its vertical displacement, i.e. the layer making contact with the face being considered. It is generally the case that the zone interface (layer) height falls midway along a face, causing both upper and lower layers to be in contact with the row of faces at that height. By using the midpoint of the face as the comparison height, this issue is consistently dealt with by assigning the layer that makes the majority of contact with each face.

A significant difference between the field and zone models is the size of time step used in the solution procedure. Zone models tend to use extremely small time steps which allow them to proceed to a solution in an explicit manner. In comparison, implicitly discretized field models such as SMARTFIRE can be solved over a wide range of time step values. Handling species flux across the hybrid interface in a summed/averaged manner can maintain conservation and ensure accurate results, but the time step discrepancy has implications for the solution of pressure within the zone model whose equations are extremely stiff with regards to this variable. A net change in enthalpy in a compartment results in a corresponding change in pressure; when this net change is caused by an enthalpy flux applying over the length of a comparatively 'large' field model time step, the resulting pressure change can be excessive. Simply using this value of zone pressure for the next iteration would result in an even greater change in values, and the solution procedure would quickly diverge to failure; this issue does not affect the accuracy of the solution, but makes reaching a solution much more difficult.

An interesting consequence of the above issue is that it differentiates between two possible configurations that define all cases applicable to treatment by the hybrid model; namely 'open' and 'closed' cases. The fundamental difference is that for the open case, the compartment or section of building being replaced by the zone model has its own vent to the outside; in other words from any point in the zone domain, it is possible to find a path to the external domain without having to first pass through any section of the CFD domain. The closed case on the other hand is characterized by the zone portion of the domain itself having no vent to the outside; any flow reaching the external domain, or any pressure release required by the zone section, would first have to pass through the hybrid interface into the CFD section. In essence, a domain having a link to the outside manifests itself as an opportunity for pressure release; this is due to the infinite nature of the 'outside', meaning that despite net flows in or out the external pressure remains at the reference pressure throughout the simulation. In this way, any simulation with an 'open' configuration circumvents the pressure/time step issue by allowing venting of any pressure build up; in effect, the value of pressure is kept within sensible limits, allowing iteration to proceed. Consequently, the hybrid implementation discussed to this point can be applied to cases of an 'open' nature.

For 'closed' cases there is no option of pressure release within the zone model domain, therefore any release must be performed through the CFD domain (via the hybrid interface) and only with respect to the time step size dictated by the CFD model. To handle these issues a custom zone model has been implemented within the hybrid model, allowing both the CFD and zone model to run over identically sized time steps. The issue of stability of the zone model over these comparatively larger time steps has been addressed by solving the zone model equations fully (to convergence) at the end of each CFD iteration, as opposed to allowing both models to iterate with the intention of reaching convergence solely at the last 
iteration of the present time step. This is in comparison to the usual 'coupling' (as used in the open case) where the zone model is also solved with respect to the current CFD iteration, but where this solution will contain an error term that tends to zero as the solution proceeds through the iterations. In essence, for the open case a residual will exist between the two models until convergence is reached (to within some tolerance); for the closed case, extra work is done in solving the zone model to ensure this residual is within the specified tolerance for each and every iteration. This clearly affects the computational effort required to solve the hybrid model, although the inherently low requirements of the zone model solution procedure results in this method remaining viable.

This pseudo-converged solution at the end of each iteration will consist of a value of pressure for the zone compartment along with the corresponding fluxes at each CFD face on the hybrid interface. Flow variables and the calculation of the zone layer sources are performed in the same manner as the open formulation [13] where variables are assigned depending on flow direction and layers assigned based on temperature and height. The mass and enthalpy sources are then used to evaluate the variables for layer $i$ as follows (subscript 0 indicates 'old' values from the previous time step),

$V_{i}=\frac{\left(m_{i, 0}+\dot{m}_{i}\right)}{\rho_{i}}$

$T_{i}=\frac{T_{i, 0} m_{i, 0}+\dot{h}_{i} / C_{P}}{m_{i, 0}+\dot{m}_{i}}+\frac{1}{C_{P}\left(m_{i, 0}+\dot{m}_{i}\right)} \cdot \frac{V_{i}}{V_{\text {room }}} \cdot(\gamma-1) \cdot\left(\sum_{j} \dot{h}_{j}\right)$

$\rho_{i}=\frac{P_{r e f}+P_{z o n e}}{R T_{i}}$

The pressure boundary condition is updated with these newly calculated values and the CFD model is run for the next iteration.

\section{TEST CASES}

As use of the hybrid model on the 'open' type of case has been demonstrated in previous work [13], the two test cases herein will focus on use of the hybrid model in a 'closed' situation. The first test case presents a situation where the zone room has two interfaces to the CFD domain; despite this it remains classified as 'closed' as it is only indirectly connected to the exterior through the second CFD room. Both cases below present instances where the zone model is in close proximity to the fire-room, testing both the stability of the hybrid implementation itself, along with its effect on the remaining CFD domain. Realistically, locations of such complex, large pressure driven flows would be reserved for the CFD model for an accurate treatment, but testing the hybrid model at its limits will provide both confidence and an idea of its limitations. In comparing between CFD and zone data, use has been made of the mass equivalency method outlined by Janssens and Tran [21] to produce approximations for the layer height and average zone temperatures based on CFD data.

\section{Test Case 1}

This case consists of three identically sized rooms (each sized $2.8 \mathrm{~m} \times 2.8 \mathrm{~m} \times 2.18 \mathrm{~m}$ ) connected via centrally located doorways $(0.74 \mathrm{~m} \times 1.83 \mathrm{~m})$ in series. The first room contains a centrally placed $62.9 \mathrm{~kW}$ heat source and is vented to the second room; the second room is then vented to the third which in turn is vented to the exterior (see Fig 2). The heat source is active for the first $60 \mathrm{~s}$ of the simulation, at which point it is turned off (i.e. $0 \mathrm{~kW}$ ). This particular value of heat release rate corresponds to one of the four fire sizes used by Steckler et al during their experiments on compartment opening flows. This case will test the hybrid model's capability in handling transient changes in simulation factors, along with its capacity to 
share the zone model with separated sections of the CFD domain. It also serves as a strong test of the hybrid implementation's stability and the effect the interface has on the CFD domain due to it being the sole means of venting available to the CFD fire room (both in the release of pressure and hot gases, and in allowing the drawing of ambient air from the exterior towards the bottom of the fire). The hybrid interface is also much closer to the fire origin than would be typically applied and this is therefore a hard test for the accuracy of the method.
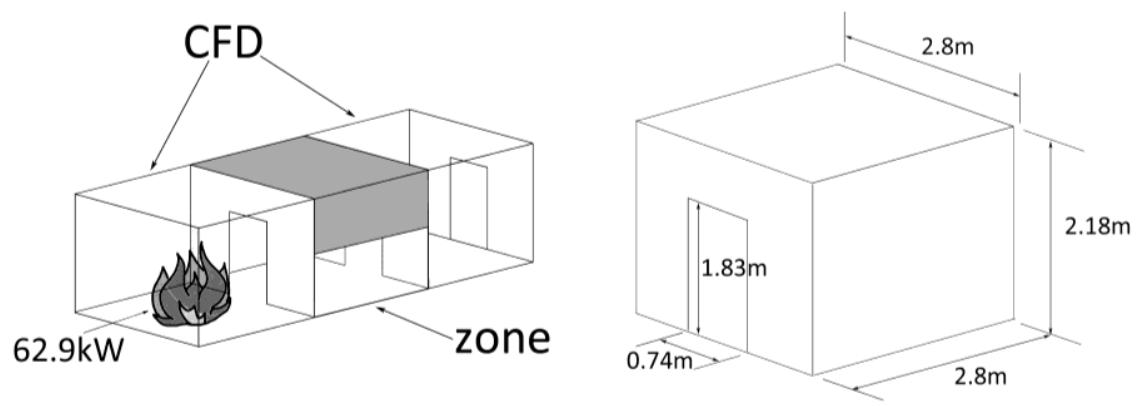

Fig. 2. Setup for Test Case 1.

The first set of comparisons is for the side rooms that remain modeled by field model in both the CFD and hybrid simulations, see Fig. 3 to Fig. 8. Results for all the CFD cases are shown at the top of each figure while results for the hybrid case are shown at the bottom of each figure.

(a)

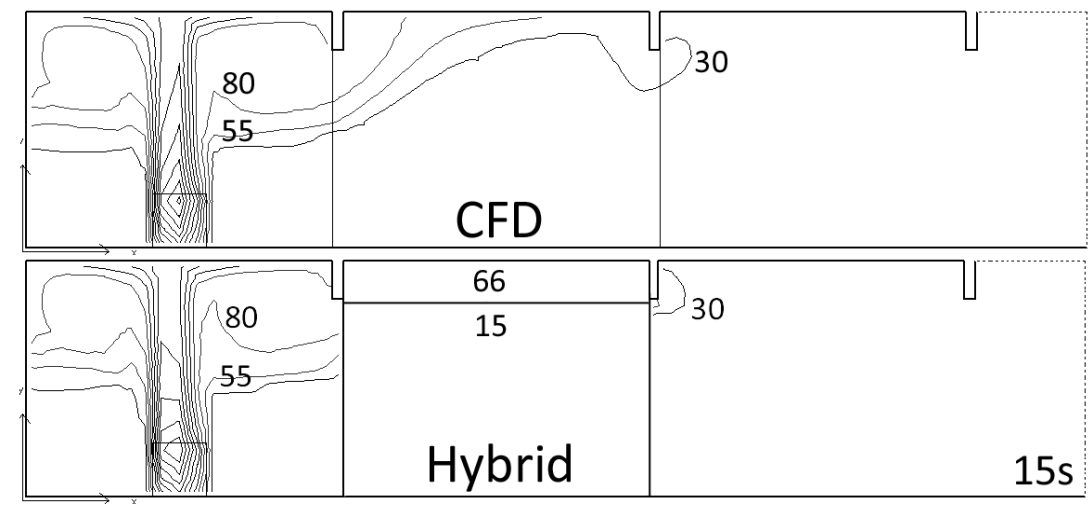

Fig. 3. Temperature $\left({ }^{\circ} \mathrm{C}\right)$ comparisons between CFD (a) and hybrid (b) at $15 \mathrm{~s}$.

(a)

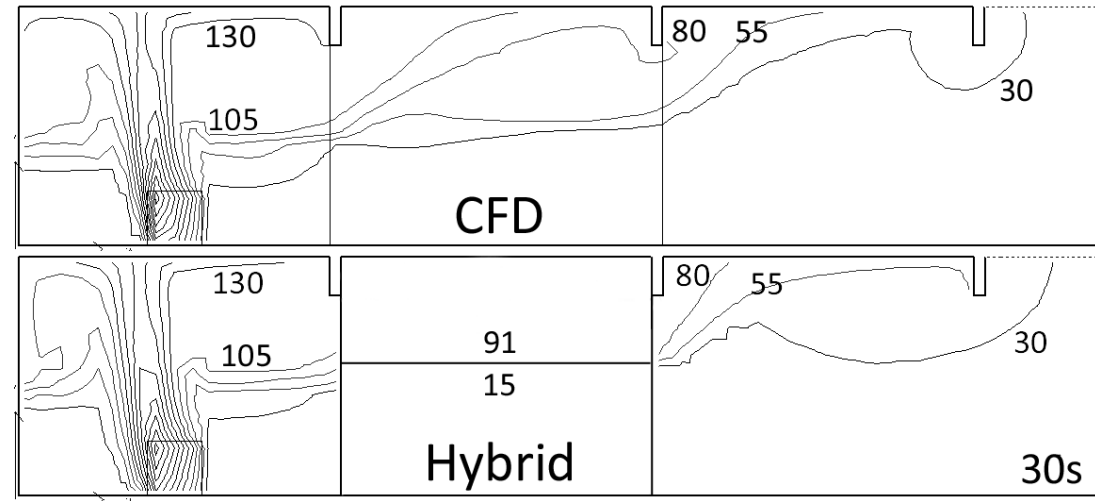

Fig. 4. Temperature $\left({ }^{\circ} \mathrm{C}\right)$ comparisons between CFD (a) and hybrid (b) at $30 \mathrm{~s}$. 
In the results for the hybrid model, the middle compartment is modeled using the zone model while the compartments at either side are modeled using the CFD approach. Within each compartment modeled using the CFD approach temperature iso-contours $\left({ }^{\circ} \mathrm{C}\right)$ are depicted while in the compartment modeled using the zone approach a visual representation of the layer height and the average upper and lower layer temperatures $\left({ }^{\circ} \mathrm{C}\right)$ are shown. Results are presented at 15, 30, 45, 60, 90 and $180 \mathrm{~s}$ after fire initiation in Fig. 3 to Fig. 8 respectively. Note that the fire is deactivated after $60 \mathrm{~s}$ and so there is no fire plume evident in Fig. 7 and Fig. 8.

(a)

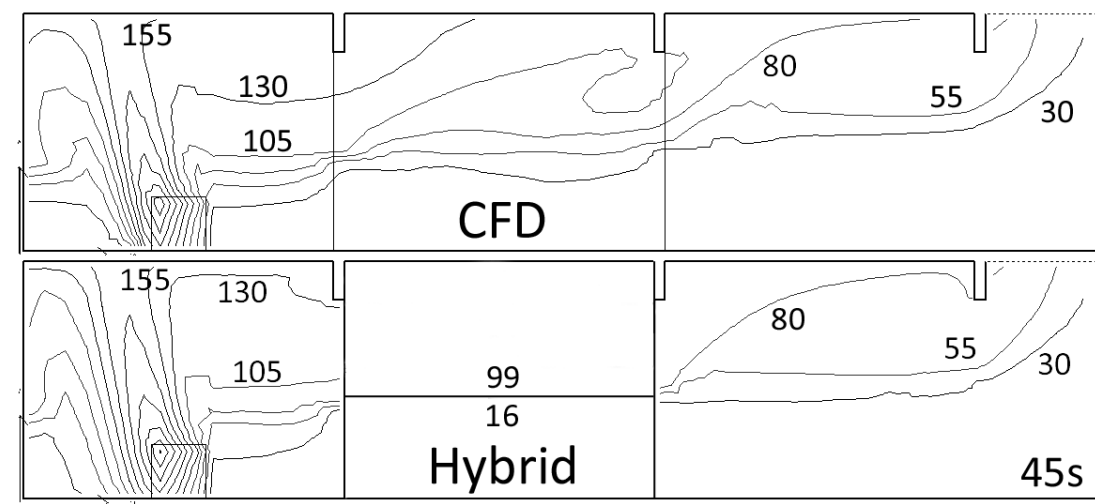

Fig. 5. Temperature $\left({ }^{\circ} \mathrm{C}\right)$ comparisons between CFD (a) and hybrid (b) at $45 \mathrm{~s}$.

(a)

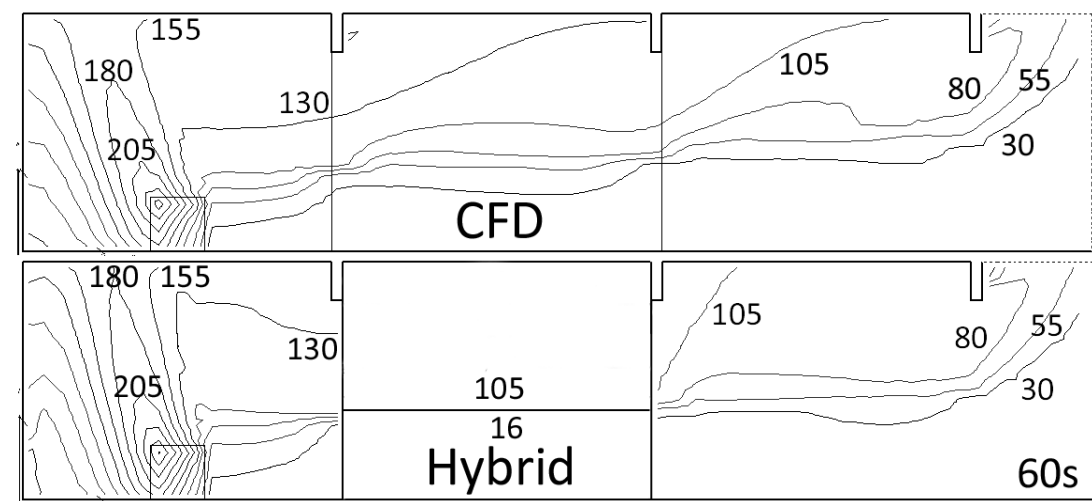

Fig. 6. Temperature $\left({ }^{\circ} \mathrm{C}\right)$ comparisons between CFD (a) and hybrid (b) at $60 \mathrm{~s}$.

(a)

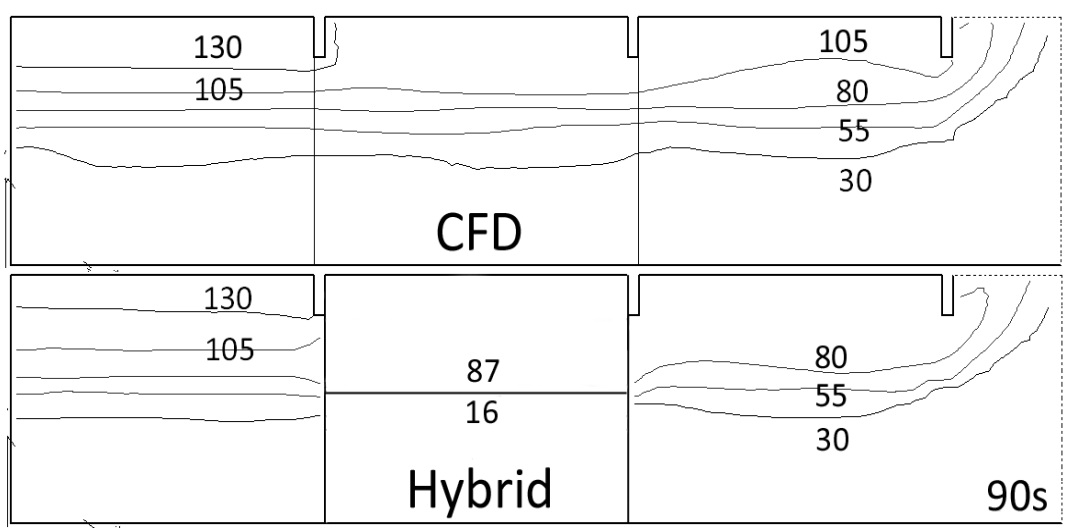

Fig. 7. Temperature $\left({ }^{\circ} \mathrm{C}\right)$ comparisons between CFD (a) and hybrid (b) at $90 \mathrm{~s}$. 
(a)

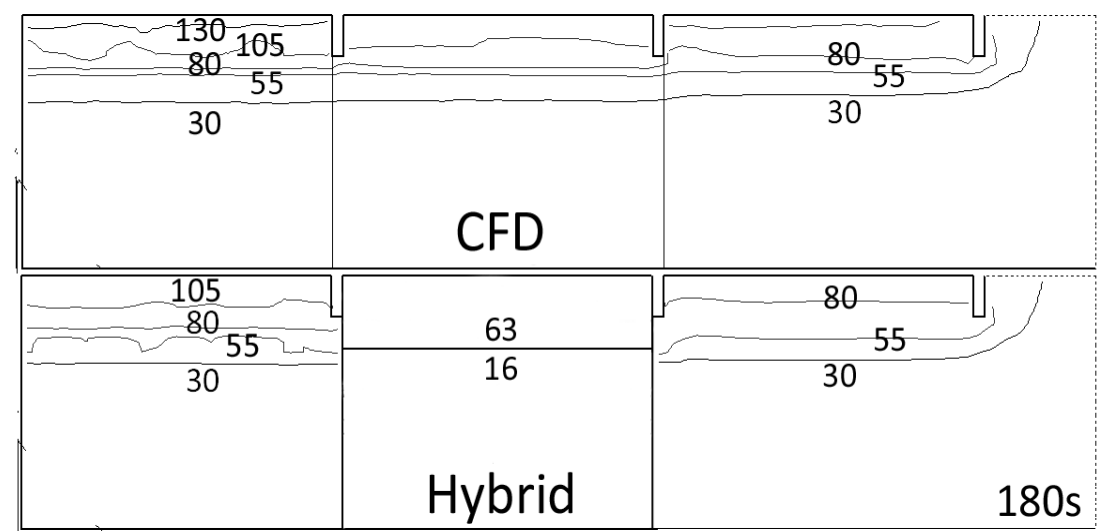

Fig. 8. Temperature $\left({ }^{\circ} \mathrm{C}\right)$ comparisons between CFD (a) and hybrid (b) at $180 \mathrm{~s}$.

From Fig. 3 to Fig. 8 it is noted that there is good agreement between the results produced by both models for both the qualitative characteristics, such as layering and the shape of the fire plume, and the quantitative values of temperature. Slight differences exist in the values of temperature observed in the compartment on the far right. This is to be expected as the upper layer temperatures at the interface that are obtained from the zone room are uniform throughout the layer, whereas the CFD simulation maintains the resolution of the temperature data. After the fire is deactivated, the rooms proceed to partially cool and again good agreement is seen between the results for this portion of the simulation, especially with regard to the heights of the layers. Figure 9 presents detailed results for the upper layer temperature and layer height for the central room that has been removed from the CFD domain and replaced with the zone model representation.

Good agreement is seen for the general trend of the upper layer temperature. Initially approximated upper layer temperatures are in good agreement, although after the fire (heat source) has been deactivated, the hybrid model appears to cool down quicker and therefore predicts lower temperatures than the CFD model. Despite this, agreement with respect to the layer height is very encouraging.

(a)

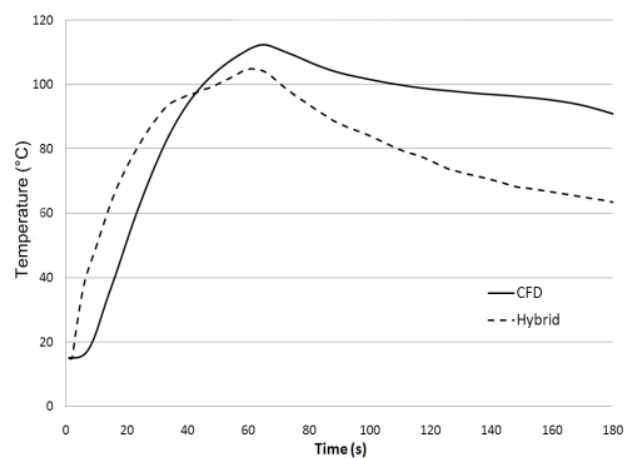

(b)

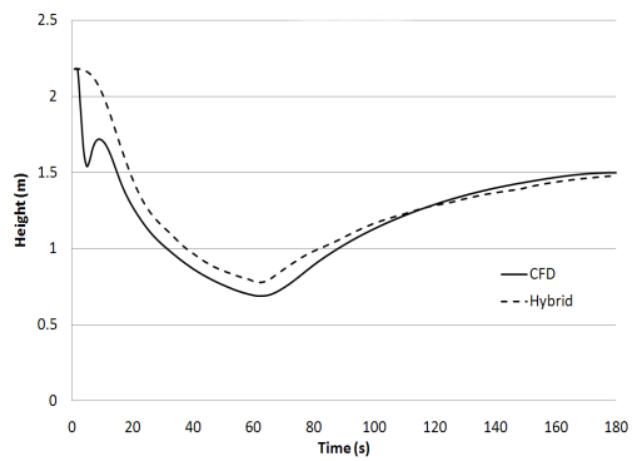

Fig. 9. Upper layer temperature $\left({ }^{\circ} \mathrm{C}\right)$ (a) and layer height (b) for the central room.

\section{Test Case 2}

The second test case consists of six identically sized compartments opening on to a common corridor that is vented to the exterior at one end (see Fig. 10). One of these compartments, situated at the closed end of the corridor, contains a centrally placed $500 \mathrm{~kW}$ heat source to represent a fire. In the hybrid model representation for this case, the remaining five compartments are modeled using the zone model approach. As a result, the hybrid model contains $56 \%$ fewer computational cells than the full CFD simulation. 


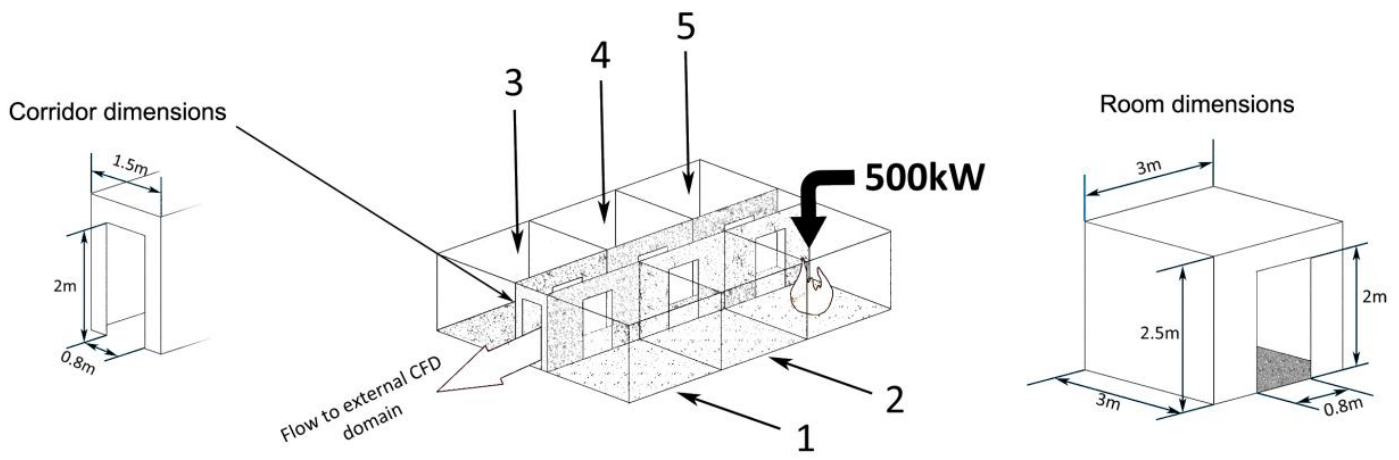

Fig. 10. Setup for Test Case 2.

The first set of results is for the sections of domain that remain modeled by the field model in both simulations (see Fig. 11 and Fig. 12). The first set of comparisons are for a cross-section through the fire room including the door leading out into the corridor (see Fig. 11) and a central cross-section along the length of the corridor itself (see Fig. 12). The contours shown are at the end of the simulation at $120 \mathrm{~s}$.

(a)

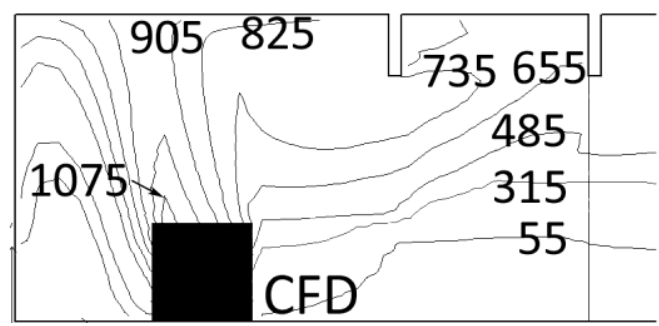

(b)

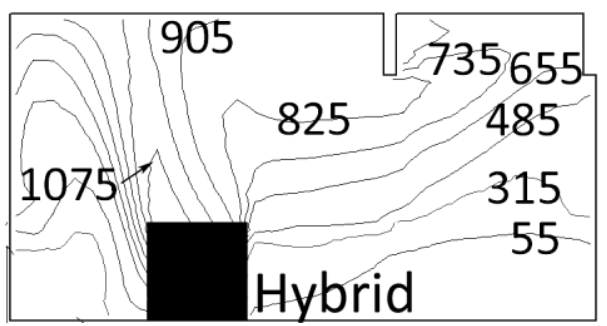

Fig. 11. Temperature $\left({ }^{\circ} \mathrm{C}\right)$ distribution within the fire room for the full CFD case (a) and the hybrid case (b) at $120 \mathrm{~s}$.

(a)

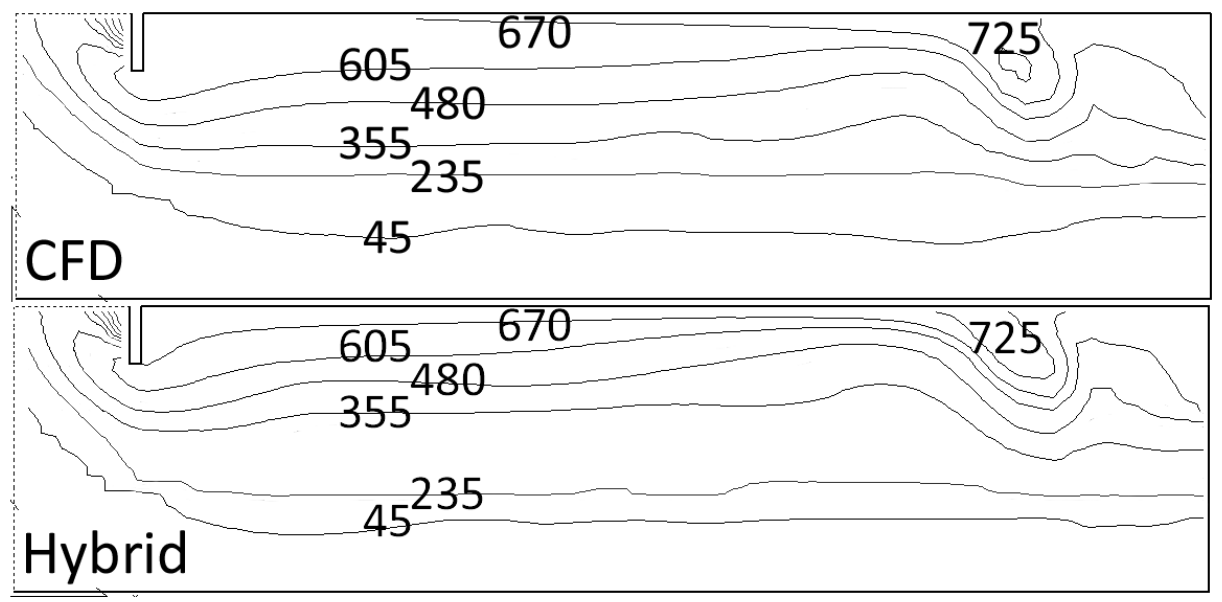

Fig. 12. Temperature $\left({ }^{\circ} \mathrm{C}\right)$ distribution within the corridor for the full CFD case (a) and the hybrid case (b) at $120 \mathrm{~s}$.

Good agreement is observed for temperatures throughout the length of the corridor (see Fig. 12). The interface height separating the ambient air and hot fire gases is also in agreement to within less than $4 \%$ of the corridor height. Again, slight banding is present in the hybrid temperatures due to the uniform layer data being applied at the interface representing each doorway. Fluxes at the external doorway at the left end of the corridor are also in agreement implying equivalent net mass balances between the two simulations.

Presented in Fig. 13 and Fig. 14 are results for two of the four rooms modeled using the zone model approach; room 1 at the external end of the corridor and room 5 opposite the fire room. Figure 13 shows 
that there is good agreement in the trends of the upper layer temperature $\left({ }^{\circ} \mathrm{C}\right)$ development between the two simulations. It is noted that the hybrid model predicts higher upper layer temperatures at the start of the simulation and lower temperatures towards the end of the simulation as compared to the CFD results.

(a)

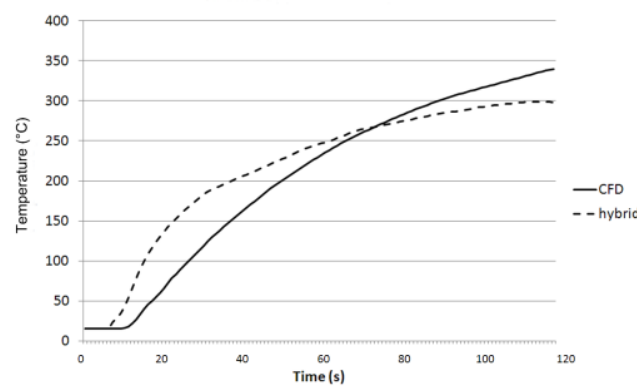

(b)

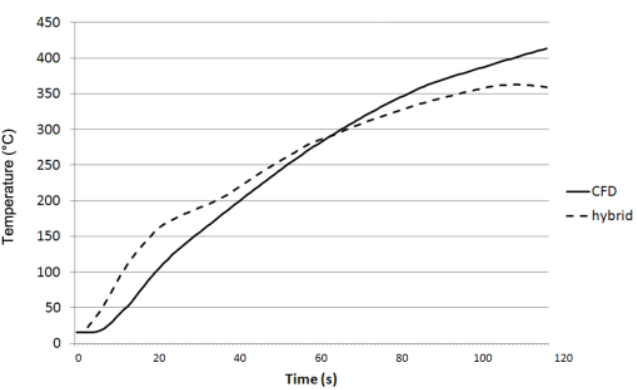

Fig. 13. Temperature comparisons for rooms 1 (a), and 5 (b), replaced by the zone model.

(a)

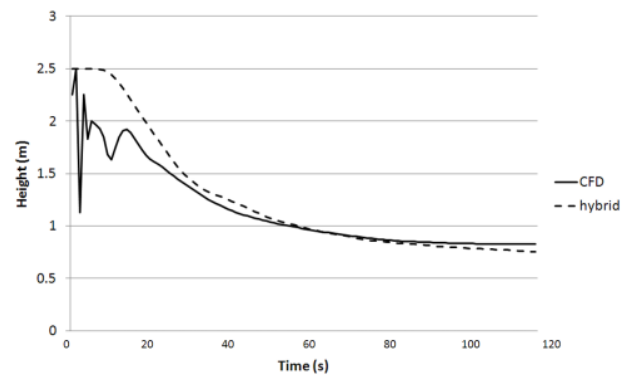

(b)

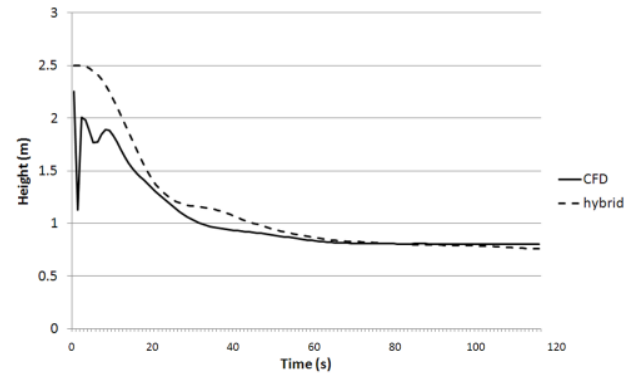

Fig. 14. Layer height comparisons for rooms 1 (a), and 5 (b), replaced by the zone model.

Figure 14 shows the layer height for the two rooms with better agreement between the two models. The hybrid model agrees well in both the size and transient development of the layers, reporting a slightly deeper layer towards the end of the simulation.

In both test cases, the method reported by Janssens and Tran [21] was used to determine average layer temperature and height based on data from a CFD fire model. This approach has produced some favourable comparisons in the two test cases, particularly with regard to layer height. It is worth noting that a positive (or negative) error in layer depth can be 'explained' somewhat by a negative (or positive) error in the layer temperature in so far as these values can vary without the total enthalpy of a layer being affected. These opposing errors are clearly apparent in Fig. 13 and Fig. 14, where an increasing disparity between the hybrid and CFD temperatures towards the end of the simulation could be explained by the fact that the hybrid layer continues to descend somewhat, as compared to that reported by the present CFD approximation. During the first $20 \mathrm{~s}$ there are fluctuations in the layer height for the CFD results; these are due to difficulties in the reduction method's ability to accurately determine the layer height when there are small temperature differences between the upper and lower layers. These fluctuations are not directly observed from the field values and is a numerical artefact of the method when a layer is not properly established. This suggests that a different method of obtaining layer averages from CFD data may produce more favourable comparisons than noted using the method of Janssens and Tran.

It is noted that for these simulations, the time required by the CFD simulation was $13 \mathrm{~h} 8 \mathrm{~min} 38 \mathrm{~s}$ while the time required by the hybrid simulation was 6 h $51 \min 29$ s, a $48 \%$ reduction in run time. Thus, by removing some $56 \%$ of the CFD solution domain, the hybrid case achieved a saving of $48 \%$ in the run time. This suggests that the hybrid model is returning an $86 \%$ computational efficiency (i.e. the model achieves a $86 \%(0.48 / 0.56)$ saving of the maximum expected savings to be made through removal of part of the domain). While this is a considerable saving in run time, by optimizing the hybrid code, it is felt that this can be further improved. However, the hybrid approach can only decrease the computational requirements of a simulation through reducing the size of the solution matrix within the CFD code. Since the run time required to perform the CFD solution procedure is not exclusively spent on operations on the system 
matrix, this percentage reduction in computational time cannot be expected to be directly proportional to the reduction of the computational mesh. It is worth noting that the total simulation time also includes time required by procedures external to the solution routine itself, such as GUI considerations or data print export, which are not affected by the hybrid implementation. It is also noted that the hybrid model can also be made to run in parallel, further reducing the run time assocated with the CFD component of the hybrid model.

\section{CONCLUSION}

A hybrid fire model has been developed that directly couples CFD fire simulation with zone modeling. The coupling is two-way with temperature and flow data passing from the field model to the zone model and from the zone model to the field model. The hybrid fire model makes use of the CFD fire model SMARTFIRE and two zone models, CFAST and an in-house zone model. The use of the hybrid model with CFAST was demonstrated in a previous publication [13] while the use of the in-house zone model within the hybrid model has been demonstrated in this paper.

Two test cases were demonstrated, one involving a situation in which a single compartment was replaced by a zone model representation and another more complex example where five compartments off a corridor were replaced by a zone model representation. In both cases the fire compartment is modeled using the CFD approach. The results demonstrate that the temperatures and layer height within the zone modeled compartments are in good agreement with the full CFD solution and the zone modeled compartments have only a minor effect on the remaining CFD domain. These results demonstrate that the hybrid model is a viable approach, and is capable of accurately replacing compartments within the field model computation domain with compartments that are modeled using the zone model approach. It is noted that making comparisons between regions modeled using the zonal and field approach are not straight forward. Disparities between the forms of data generated by the respective models means that conversion of some form is required before equivalent comparisons can be made. Essentially, this means that it is necessary to reduce the resolution of the CFD data to that equivalent to a two layer uniform zone; clearly this must be performed through averaging of some kind. The choice of an appropriate method to use is not trivial and small differences in the averaged results can be expected based on the averaging scheme selected. It is thus difficult to be precise concerning the level of agreement achieved between the zone results and the CFD results.

The reduction in run time achieved by the hybrid approach represents $86 \%$ of the saving that could be expected for the associated reduction in the CFD computational domain. While the savings in computational time achieved by the hybrid model cannot be expected to scale directly to the reduction in the CFD computational domain, further improvements are expected through optimisation of the hybrid code. Further work is required to improve both the accuracy and convergence of the hybrid model and to test the approach on more complex cases. In addition, further developments of the hybrid approach will include radiation handling and transportation of species.

\section{ACKNOWLEDGEMNT}

Mr. Burton gratefully acknowledges the financial support of FSEG through the University of Greenwich $\mathrm{PhD}$ bursary programme. Project FIREPROOF (contract 218761) is funded under the European Union Framework 7 Transport initiative.

\section{REFERENCES}

[1] Yan, Z., and Holmstedt, G., "Investigation of the dance hall fire in Gothenburg, October 1998 - a comparison between human observation and CFD simulation," Interflam 2001, Interscience Communications, 2001, pp. 951-963.

[2] Luo, M., and Beck. V., "Flashover fires in a full scale building: prediction and experiment," Interflam 1996, Interscience Communications, 1996, pp. 361-370.

[3] Wang, Z., Jia, F., Galea, E.R., Patel, M.K., and Ewer, J., (2001) Simulating one of the CIB W14 round robin test cases using the SMARTFIRE fire field model, Fire Safety Journal 36(7): 661-677, http://dx.doi.org/10.1016/S0379-7112(01)00018-2. 
[4] Jia, F., Patel M.K., Galea E.R., Grandison, A., and Ewer, J., (2006) CFD Fire Simulation of the Swissair Flight 111 In-flight Fire - Part II: Fire Spread within the Simulated Area, The Aeronautical Journal of the Royal Aeronautical Society 110(1107): 303-314.

[5] Chow, W.K., (1995) A comparison of the use of fire zone and field models for simulating atrium smoke-filling processes, Fire Safety Journal 25(4): 337-353, http://dx.doi.org/10.1016/03797112(96)00001-X.

[6] Grandison, A.J., Galea, E.R., Patel, M.K., and Ewer, J., (2003) The development of parallel implementation for a CFD based fire model utilising conventional office based PCs, Journal of Applied Fire Science 12(2): 137-157, http://dx.doi.org/10.2190/AGH5-EXUR-J110-HPHE.

[7] Hurst-Clark, N.M., Ewer, J.A.C., Grandison, A.J., and Galea, E.R., "Group Solvers: A Means of Reducing Run-Times and Memory Overheads for CFD Based Fire Simulation Software," Interflam 2004, Interscience Communications, 2004, pp. 659-664.

[8] Xu, T., Wang, J., and Fan, W., "A new method of modelling of a fire - combination of field modelling and zone modelling," Symposium on Engineering Thermophysics, ZhenJiang, China, 1991, pp. 33-45.

[9] Wang, J., and Fan, W., (1996) Numerical simulation of fire process of multi-rooms, J Univ Sci Technol 26: 204-209.

[10] Hua, J., Wang, J., and Kumar, K., (2005) Development of a hybrid field and zone model for fire smoke propagation simulation in buildings, Fire Safety Journal 40(2): 99-119, http://dx.doi.org/10.1016/j.firesaf.2004.09.005.

[11] Jones, W.W., Peacock, R.D., Forney, G.P., and Reneke, P.A., "CFAST - Consolidated Model of Fire Growth and Smoke Transport (Version 6), Technical Reference Guide," National Institute of Standards and Technology Special Publication 1026, Gaithersburg, MD, 2009.

[12] Wang, Z., Jia, F., and Galea, E., (2007) Predicting toxic gas concentrations resulting from enclosure fires using local equivalence ratio concept linked to fire field models, Fire and Materials 31(1): 27-51, http://dx.doi.org/10.1002/fam.924.

[13] Burton, D.J., Grandison, A.J., Patel, M.K., Galea, E.R., and Ewer, J.C., "Introducing a hybrid field/zone fire modelling approach for fire simulation," Interflam 2007, Interscience Communications, 2007, pp. 1491-1497.

[14] Quintiere, J., "Growth of fires in building compartments," American Society for Testing and Materials, ASTM STP 614, Philadelphia, PA, 1977.

[15] Pape, R., Waterman, T.E., and Eichler, T.V., "Development of a fire in a room from ignition to full room involvement - RFIRES," National Bureau of Standards, NBS-GCR-81-301, Washington, 1981.

[16] Galea, E., (1989) On the Field Modelling Approach to the Simulation of Enclosure Fires, Journal of Fire Protection Engineering 1(1): 11-22, http://dx.doi.org/10.1177/104239158900100103.

[17] Li, Y.F., and Chow, W.K., (2004) Modelling of water mist fire suppression systems by a one-zone model, Combustion Theory and Modelling 8(3): 567-592, http://dx.doi.org/10.1088/1364$\underline{7830 / 8 / 3 / 008 .}$.

[18] Konecki, M., and Pólka, M., (2009) Extension of the fire zone model with some detailed mass and heat transfer mechanisms, Journal of Applied Sciences Research 5(2): 212-220.

[19] Chen, X., Yang, L., Deng, Z., and Fan, W., (2004) A multi-layer zone model for predicting temperature distribution in a fire room, Progress in Natural Science 14(6): 536-540, http://dx.doi.org/10.1080/10020070412331343901.

[20] Patankar, S.V., Numerical Heat Transfer and Fluid Flow, McGraw-Hill, New York, 1980.

[21] Janssens, M. and Tran, H., (1992) Data Reduction of Room Tests for Zone Model Validation, Journal of Fire Sciences 10(6): 528-555, http://dx.doi.org/10.1177/073490419201000604. 